\title{
TAZ is overexpressed in prostate cancers and regulates the proliferation, migration and apoptosis of prostate cancer PC3 cells
}

\author{
MENGYUAN LIN ${ }^{1,2}$, CHAOZHI BU ${ }^{1,2}$, QINGWEN HE ${ }^{1,2}$, JIAYU GU $^{3}$, \\ HONGHUA WANG ${ }^{1,2}$, NINGHAN FENG ${ }^{3}$ and SHI-WEN JIANG ${ }^{1,2}$
}

\author{
${ }^{1}$ Joint Laboratory of SKLRM (State Key Laboratory of Reproductive Medicine), The Affiliated Wuxi Maternity and \\ Child Health Care Hospital of Nanjing Medical University; ${ }^{2}$ Research Institute for Reproductive Health and \\ Genetic Diseases, Reproductive Center, The Affiliated Wuxi Maternity and Child Care Health Hospital of \\ Nanjing Medical University, Wuxi; ${ }^{3}$ Department of Urology, The Affiliated Wuxi No. 2 People's \\ Hospital of Nanjing Medical University, Wuxi, Jiangsu 214002, P.R. China
}

Received August 31,2019; Accepted April 14, 2020

DOI: $10.3892 / o r .2020 .7616$

\begin{abstract}
TAZ (transcriptional coactivator with PDZ-binding motif), which is also known as WW domain-containing transcription regulator 1 (WWTR1), a downstream effector of the Hippo pathway, has been reported to regulate cancer cell proliferation, migration and apoptosis by acting as a transcriptional coactivator. However, the function of TAZ in prostate cancer cells has not been investigated. In the present study, TAZ expression in prostate cancer (PCa) and benign prostatic hyperplasia tissues, $\mathrm{PCa}$ cell lines, and normal prostate epithelial cells was determined with the use of immunohistochemistry. TAZ was knocked down by shRNA in the PC 3 cells, a prostate cancer cell line, and cell viability and migration assays were performed to determine the biological functions of TAZ. A mouse subcutaneous xenograft model was used to determine the in vivo effects of TAZ knockdown on tumor growth. We demonstrated that TAZ is overexpressed in $\mathrm{PCa}$ tissues, and the expression levels were found to be positively correlated with the Gleason scores of cancer grade. Moreover, TAZ knockdown inhibited PC3 cell proliferation, reduced cell migration, and induced apoptosis. Further experiments demonstrated that TAZ knockdown may lead to PC3 cell apoptosis through the exogenous apoptotic pathway by
\end{abstract}

Correspondence to: Professor Shi-Wen Jiang, Research Institute for Reproductive Health and Genetic Diseases, Reproductive Center, The Affiliated Wuxi Maternity and Child Care Health Hospital of Nanjing Medical University, 48 Huaishu Lane, Liangxi, Wuxi, Jiangsu 214002, P.R. China

E-mail: jiangsw137@163.com

Professor Ninghan Feng, Department of Urology, The Affiliated Wuxi No. 2 People's Hospital of Nanjing Medical University, Wuxi, 68 Zhongshan Road, Jiangsu 214002, P.R. China

E-mail: fnh888@njmu.edu.cn

Key words: TAZ, prostate cancer, PC3 cells, proliferation, migration, apoptosis inducing the expression and cleavage of caspase- 4 and -7 . In the tumor xenograft model, TAZ knockdown led to a decreased tumor growth rate. Taken together, the experimental results indicate that TAZ plays a significant role in the proliferation, migration and apoptosis of prostate cancer cells. TAZ could be a useful biomarker for PCa diagnosis/prognosis, and it could be a potential target for the treatment of prostate cancers.

\section{Introduction}

Prostate cancer ( $\mathrm{PCa}$ ) is a commonly diagnosed malignancy, and it is the second leading cause of cancer-related deaths among American males $(1,2)$. While early detection and adjuvant therapy have led to the reduced death rate of PCa patients in the past decade worldwide $(1,3)$, management of late-stage $\mathrm{PCa}$ cases remains a challenge. A better understanding of the pathologic mechanisms underlying the initiation, progression, and metastasis of prostate cancer is required for future improvement in the detection, prevention, and treatment of this common disease. TAZ (transcriptional coactivator with PDZ-binding motif), which is also known as WW domain-containing transcription regulator 1 (WWTR1) (4), shares amino acid sequence homology with the WW domain of the Yes-associated protein (YAP) in amino acid sequences (4). TAZ and YAP form a protein complex that shuttles between the cytoplasm and the nucleus $(4,5)$. By interacting with other transcriptional factors such as the TEAD domain family (TEAD1, TEAD2, TEAD3, and TEAD4), the complex serves as a transcriptional coactivator that regulates gene transcription (5). It was reported that TAZ and YAP are controlled by the Hippo pathway by two mechanisms. The Hippo pathway activates the core kinase cassette, leading to repression of TAZ and YAP functions. Alternatively, LAST, a component of the Hippo pathway, interacts with TAZ and YAP, sequestering them at cell-cell junctions and preventing their nuclear translocation $(6,7)$.

Overexpression of YAP and TAZ as a result of gene amplification and epigenetic modulation has been detected in a variety of malignancies such as breast, liver, lung, gastric, ovarian and colorectal tumors (8-14). Increased levels of TAZ 
in tumor tissues were associated with poor prognosis of retinoblastoma and non-small cell lung carcinoma $(15,16)$. YAP- and TAZ-mediated gene transcription is important for the development and sustainability of malignant behaviors of cancer cells $(12,14,17)$. Chan et al reported that forced overexpression of TAZ in a breast cell line (MCF10A cells) induced morphologic changes and promoted cell migration and invasion (13), whereas knockdown of TAZ in breast cancer MCF7 and Hs578T cells suppressed cell migration and invasion. TAZ knockdown in MCF7 cells also led to an inhibition of cell proliferation in soft agar and the cancer growth rate in a mouse subcutaneous xenograft model (13). Lysophosphatidic acid (LPA) has been reported to contribute to the tumorigenesis and metastasis by promoting the proliferation, migration, and invasion of cancer cells (18). Jeong et al reported that treatment with LPA increased the motility of an ovarian epithelial cancer cell line (R182) in the wound scratch assay (11). Interestingly, knockdown of TAZ expression using a TAZ-specific siRNA resulted in the abrogation of LPA-stimulated cell mobility in the wound scratch assay (11). These results suggest that TAZ may play an important role in the regulation of cell anchorage-independent growth and migration. The function of TAZ in prostate cancer has not been investigated. In the present study, we examined the role of TAZ in the proliferation, migration and apoptosis of PC3 prostate cancer cell line and the effect of TAZ knockdown on tumor growth in the mouse xenograft model.

\section{Materials and methods}

Collection of clinical specimens. This study was approved by the Human Research Ethics Committee of the Affiliated Wuxi No. 2 People's Hospital of Nanjing Medical University (Wuxi, Jiangsu, China) (approval no. 20170719). All participants signed informed consent prior to enrollment. This study was conducted according to the principles of the Declaration of Helsinki. Patients were diagnosed following the Guidelines for Diagnosis and Treatment of Urology in China (2014) (19). Thirty-three patients (aged between 63-78 years, mean age 69.4 years) with benign prostatic hyperplasia (BPH), 23 patients (aged between 56-79 years, mean age 67.3 years) with low Gleason scores (L-GL) and 25 (aged between 63-81 years, mean age 71.3 years) patients with high Gleason scores (H-GL) were included in the study.

Immunohistochemistry (IHC). Human prostate cancer and benign prostate hyperplasia tissues obtained from surgery fixed and embedded by routine procedures were retrieved from the Department of Pathology at the Affiliated Wuxi No. 2 People's Hospital of Nanjing Medical University (from September 2017 to May 2018). Tissue sections were deparaffinized three times in fresh xylene for 5 min each time, before they were rehydrated sequentially with $100,95,80$ and $75 \%$ ethanol/PBS solutions for $5 \mathrm{~min}$ at each step. Paraffin-embedded tissues were cut into $5 \mu \mathrm{m}$ thick sections. Antigen retrieval was performed with a 2100-Retriever (PickCell Laboratories BV, Prestige Medical, Ltd.) for $12 \mathrm{~min}$ in sodium citrate buffer ( $\mathrm{pH}$ 6.0). The slides were quenched with $0.6 \% \mathrm{H}_{2} \mathrm{O}_{2}$ for 20 min. After rinsing with PBS, the sections were blocked in PBS containing $5 \%$ goat serum and $2 \%$ bovine serum albumin for $2 \mathrm{~h}$ at room temperature, and they were subsequently incubated overnight with the primary antibody (dilution 1:1,000, anti-TAZ, Abcam, USA; cat. no. ab84927) at $4^{\circ} \mathrm{C}$. The slides were washed with PBS containing $0.1 \%$ Tween 20 , and then they were incubated with a secondary antibody (dilution 1:2,000, anti-rabbit, Cell Signaling Technology, Inc. (CST), China; cat. no. 8114) for $2 \mathrm{~h}$. After extensive washing, the slides were incubated with Vectastain ABC reagent for $60 \mathrm{~min}$. A diaminobenzidine tetrahydrochloride peroxidase substrate (Dako Envision System K 1395; Dako) was applied to the slides for 3 to $5 \mathrm{~min}$ in the dark, and reactions were terminated by washing in PBS. The slides were observed under a microscope (Olympus BX53; Olympus Corp.) with magnification $\mathrm{x} 200$, and images were captured and analyzed. Positive cells were counted with the use of ImageJ software (National Institutes of Health, Germany; v1.8.0), and the number of positive cells were calculated and compared.

Cell culture. Prostate carcinoma cell lines (PC3, DU-145, 22RV1 and LNCaP cells) were purchased from the American Type Culture Collection (ATCC). The normal human prostate epithelial cell line RWPE-1 was obtained from Professor Chun Lu of Nanjing Medical University. PC3, DU-145 and LNCaP cells were maintained in RPMI-1640 medium, and 22RV1 cells were maintained in DMEM/F-12. All media were supplemented with $10 \%$ fetal bovine serum (FBS), $100 \mathrm{U} / \mathrm{ml}$ penicillin, and $100 \mu \mathrm{g} / \mathrm{ml}$ streptomycin. Cell cultures were maintained at $37^{\circ} \mathrm{C}$ in a $5 \% \mathrm{CO}_{2}$ atmosphere.

Cell transfection with short hairpin RNA (shRNA). PC3 cells grown to $70 \%$ confluence were transfected with shRNA plasmid DNA using jetPRIME transfection reagent (Polyplus) according to the manufacturer's instructions. The shRNA duplexes were designed by GenePharma as follows: TAZ-1, GGCAGTATCCCAGCCAAATCT; TAZ-2, GGCTCATGA GTATGCCCAATG; TAZ-3, GCAGCAGAAACTGCG GCTTCA; TAZ-4, GCTCAGATCCTTTCCTCAATG; NC, non-specific control, contains the scrambled sequences with the same length of TAZ-specific oligonucleotides: TTCTCC GAACGTGTCACGTCT. The efficacy of plasmid constructs was verified by real-time PCR and western blot analysis after cell transfection.

Western blot analysis. Cells $\left(1 \times 10^{6}\right)$ were seeded in 6-well plates and grown to 70-80\% confluency before they were lysed with whole lysis buffer (50 mM HEPES, $150 \mathrm{mM}$ $\mathrm{NaCl}, 1 \mathrm{mM}$ EGTA, $10 \mathrm{mM}$ sodium pyrophosphate, $1.5 \mathrm{mM}$ $\mathrm{MgCl}_{2}, 100 \mathrm{mM}$ sodium fluoride, $10 \%$ glycerol, and $1 \%$ Triton $\mathrm{X}-100)$. Cell lysates were centrifuged $(12,000 \mathrm{x} \mathrm{g})$ for $30 \mathrm{~min}$ at $4^{\circ} \mathrm{C}$ and supernatants were used for western blotting. Cell proteins were resolved by a $10 \%$ SDS-PAGE gel, transferred onto nitrocellulose membranes (GE Healthcare Life Sciences) and stained with $0.1 \%$ Ponceau S solution (Sigma-Aldrich; Merck $\mathrm{KGaA}$ ) to assess transfer and equal loading of samples $(50 \mu \mathrm{g})$. After blocking in 5\% nonfat milk for $30 \mathrm{~min}$, the membranes were blotted with the following primary antibodies overnight at $4^{\circ} \mathrm{C}$, including anti- $\beta$-actin (dilution 1:1,000, Abcam; cat. no. 8226), anti-TAZ (dilution 1:2,000, Abcam; cat. no. ab84927), anti-caspase-4 (dilution 1:2,000, Abcam; cat. no. 25898), anti-caspase-7 (dilution 1:2,000, Abcam; cat. no. 69540), and anti-caspase-9 (dilution 1:2,000, Abcam; cat. no. 202068). After washing, the membranes were 
incubated with horseradish peroxidase-conjugated secondary antibodies (dilution 1:2,000, anti-rabbit and anti-mouse, Cell Signaling Technology, Inc.; cat. nos 14708 and 14709) for $30 \mathrm{~min}$ at room temperature. An enhanced chemiluminescence Western blotting system (GE Healthcare Life Sciences) was applied for image development following the manufacturer's instructions.

RNA isolation and real-time quantitative PCR. Total RNA was isolated using TRIzol reagent (Invitrogen; Thermo Fisher Scientific, Inc.). One microgram of total RNA in a volume of $20 \mu \mathrm{l}$ was reverse transcribed using the SuperScript III reagent (Thermo Fisher Scientific, Inc.). Real-time PCR was performed with a 7500 PCR machine (Applied Biosystems, USA) in a total volume of $20 \mu \mathrm{l}$, which consisted of $10 \mu \mathrm{l}$ of $2 \mathrm{X}$ SYBR Master Mix (Applied Biosystems), 10 pM of forward and reverse primers (COSMO Genetech) and diluted cDNA. The PCR conditions were as follows: Initial denaturation at $95^{\circ} \mathrm{C}$ for $5 \mathrm{~min}$, followed by 40 cycles of denaturation at $95^{\circ} \mathrm{C}$ for $10 \mathrm{sec}$ and amplification at $59^{\circ} \mathrm{C}$ for $45 \mathrm{sec}$. The primer sequences were as follows: TAZ forward: TCAACAGCTCAA CTTTCGGG; TAZ reverse: TTGGCTGACAAATCCCGA $\mathrm{CC} ; \beta$-actin forward: CATGTACGTTGCTATCCAGGC; and $\beta$-actin reverse: CTCCTTAATGTCACGCACGAT. Relative mRNA levels of the target genes were normalized to those of the $\beta$-actin gene, which served as an internal reference, and the relative changes in the mRNA levels were calculated by the $2^{-\Delta \Delta \mathrm{Cq}}$ method (20).

MTT cell viability assay. PC3 cells were seeded in 96-well plates at a density of $1 \times 10^{3}$ cells per well. Ten microliters of MTT dye (Sigma-Aldrich; Merck KGaA) was added to each well, and incubated with the cell culture for $4 \mathrm{~h}$. Formazan dissolved in DMSO (100 $\mu$ l) was added to each well, and the absorbance was measured by a microplate reader at $490 \mathrm{~nm}$ and recorded.

Wound scratch assay. PC3 cells $\left(1 \times 10^{6}\right)$ were seeded in 6-well plates and then were transfected with shRNA plasmid DNA. Artificial wounds were generated in the cell cultures using pipette tips $(4 \mathrm{~cm})$ and images were taken under a microscope and recorded. Wound healing was visualized at 0,24 and $48 \mathrm{~h}$. For each group, 3 artificial wounds were photographed at different time points and the relative cell migration rates were calculated.

Flow cytometric analysis. For cell apoptosis assay, PC3 $\left(1 \times 10^{6}\right)$ and DU-145 $\left(1 \times 10^{6}\right)$ cells were seeded in 6-well plates and then were transfected with the indicated shRNA plasmid DNA. Floating and adherent PC3 and DU-145 cells were collected, washed, and resuspended in $500 \mu \mathrm{l}$ of staining buffer containing $5 \mu \mathrm{l}$ of Annexin-V FLUOS and $5 \mu \mathrm{l}$ of propidium iodide (PI) (BD Biosciences). Following incubation on ice in the dark for $15 \mathrm{~min}$, cells were examined by flow cytometry (BD FACS Aria III, BD Biosciences). The fluorescence of FITC and PI was measured in the BV421 channel (405 nm) and PE channel $(561 \mathrm{~nm})$, respectively.

In vivo xenograft model. The usage of mice for the xenograft experiment was approved by the Institutional Animal Ethics
Committee of Jiangnan University. In total, 5 athymic male mice (weight, 16-20 g; age, 4-6 weeks) were purchased from the Animal Center of the Chinese Academy of Science (Shanghai, China). Animal xenograft experiments were performed following the ARRIVE Guidelines (https://www. nc3rs.org.uk/arrive-guidelines). Mice were housed at the animal facility of the Laboratory Animal Center of Jiangnan University. The experimental conditions of the mice included: A specific pathogen-free environment, $12 \mathrm{~h}$ light/dark cycle, and free access to food and water. PC3 cells were stably transfected with shRNA vectors and then were harvested by trypsin digestion. In order to establish a subcutaneous tumor model, five million cultured PC 3 cells in $200 \mu 1$ of volume were inoculated subcutaneously into the mice. Five mice were used in each of the experimental or control group. The tumor volume (V) was estimated every 2 days using the equation: $\mathrm{V}=0.5 \times \mathrm{a} \mathrm{x} \mathrm{b}^{2}$ (a, length; $\mathrm{b}$, width). Sixteen days after injection, the mice were anesthetized intraperitoneally with a $1 \%$ sodium pentobarbital solution as $45 \mathrm{mg} / \mathrm{kg}$, before cervical dislocation was used for sacrifice. Throughout the experiments, tumor volume was monitored to ensure that the tumor diameter would not exceed $15 \mathrm{~mm}$.

Statistical analyses. Quantitative data are expressed as the mean \pm SD. Statistical analyses were performed using SPSS Statistics 20.0 (IBM Corp.). For comparison among multiple groups, ANOVA tests were first performed and if the P-value was less than 0.05 , Tukey test was subsequently conducted for one-to-one comparison between different groups. Student's t-test was performed when two groups were compared. Spearman regression was perform to analyze the correlation between TAZ expression and Gleason scores. $\mathrm{P}<0.05$ was considered to indicate a statistically significant difference.

\section{Results}

Increased TAZ expression in prostate cancer tissues. Samples from 33 patients with benign prostatic hyperplasia $(\mathrm{BPH})$, 23 patients with low Gleason scores $(\leq 6, \mathrm{~L}-\mathrm{GL})$ and 25 patients with high Gleason scores ( $>6, \mathrm{H}-\mathrm{GL})$ were collected and examined for TAZ expression. We first compared TAZ mRNA expression levels in BPH, well differentiated, and poorly differentiated prostate cancer tissues. The results of real-time PCR indicated significantly increased TAZ mRNA levels in the L-GL and H-GL groups compared to that of the BPH group (Fig. 1A). Western blot analysis verified the increased TAZ protein expression in cancer tissues (Fig. 1B). Fig. 1C shows the immunohistochemistry (IHC) results of two representative samples from each group. TAZ was mostly expressed in glandular epithelial cells. While weak staining was detected in BPH samples, strong staining was observed in the L-GL and H-GL specimens. Semi-quantitative analysis confirmed that while both L-GL and H-GL groups contained more TAZ-positive cells compared to the BPH group, $\mathrm{H}-\mathrm{GL}$ tissues contained more TAZ-positive cells than L-GL tissues (Fig. 1D; upper graph). The Gleason score system was applied to provide an objective measure of the malignant characteristics of cancers. Regression analysis showed that TAZ expression positivity was quantitatively and positively correlated with Gleason scores in the L-GL and H-GL groups 
A

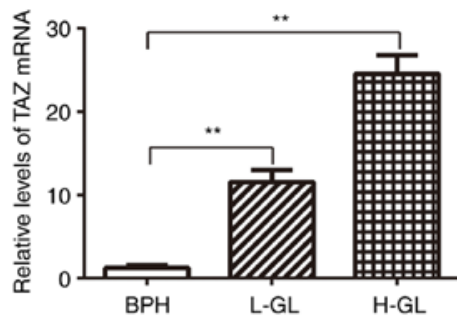

C

$\mathrm{BPH}$

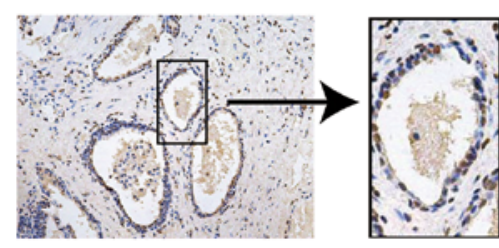

L-GL

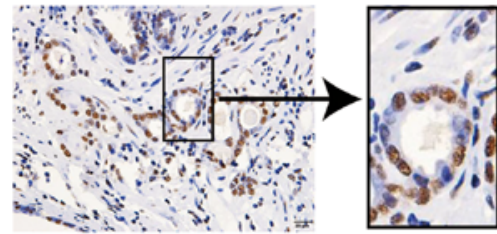

H-GL

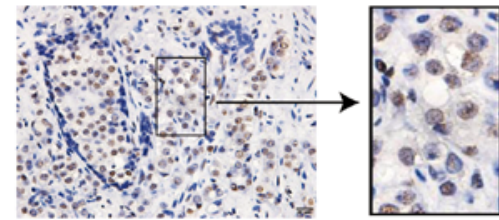

B

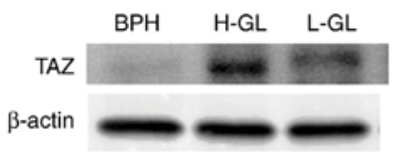

D
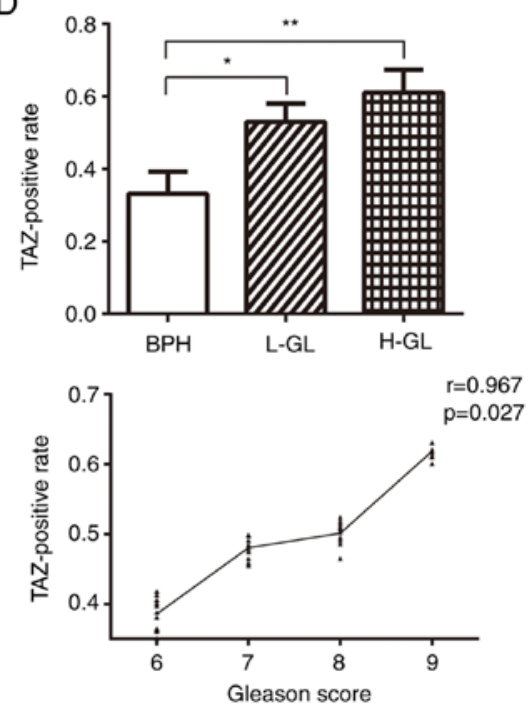

Figure 1. TAZ expression levels in BPH, L-GL and H-GL tissues. (A) Results of real-time quantitative PCR, show significantly increased TAZ mRNA levels in H-GL and L-GL tissues compared with those in BPH tissues. (B) Representative results of western blot analysis show TAZ overexpression in H-GL and L-GL tissues. (C) Representative images of immunohistochemical analysis of BPH, L-GL and H-GL tissues with the use of TAZ-specific antibodies (magnification $\mathrm{x} 200$ ). Note the significantly higher TAZ positive rate in $\mathrm{H}-\mathrm{GL}(\mathrm{N}=25)$ and $\mathrm{L}-\mathrm{GL}(\mathrm{N}=23)$ when compared to BPH (N=33) cases. (D) Upper panel: Immunohistochemistry images were analyzed with the use of ImageJ software and a significantly higher TAZ-positive cell rate was found in cancer tissues when compared to this rate in the control tissues. Lower panel: Regression analysis showed that TAZ expression positivity was positively correlated with the Gleason scores in the L-GL and H-GL groups. ${ }^{*} \mathrm{P} \leq 0.05$, and ${ }^{* *} \mathrm{P} \leq 0.01$. TAZ, transcriptional coactivator with PDZ-binding motif; BPH, benign prostatic hyperplasia; H-GL, high Gleason score; L-GL, low Gleason score.

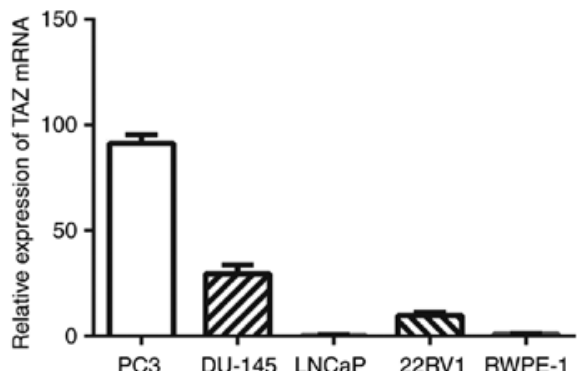

B

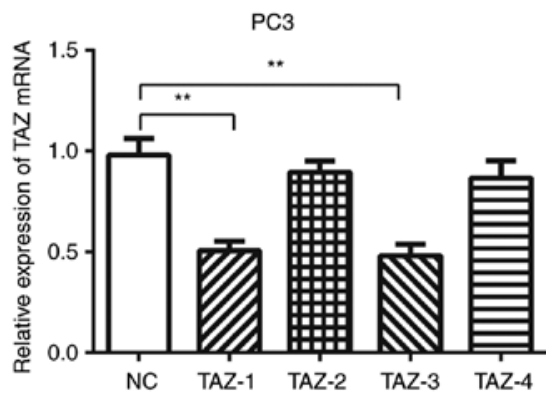

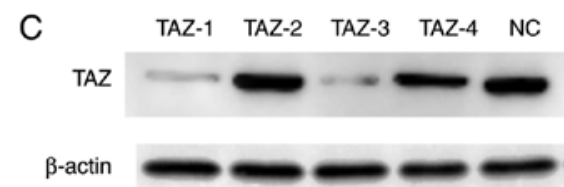

Figure 2. TAZ expression levels in different prostate cancer cell lines and efficiency of TAZ knockdown in PC3 cells. (A) Real-time PCR results demonstrating different TAZ mRNA levels in PC3, DU-145, LNCaP, 22RV1 and RWPE-1 cell lines. (B) TAZ mRNA levels in PC3 cells $48 \mathrm{~h}$ after transfection with 4 short hairpin RNA species. $\beta$-actin mRNA levels were used as an internal reference. Compared to the nonspecific control (NC) levels, TAZ-1 and TAZ-3 transfection efficiently inhibited TAZ mRNA expression. (C) Western blot analysis confirmed the high efficiency of TAZ-1 and TAZ-3 in the silencing of TAZ in PC3 cells. ${ }^{* *} \mathrm{P} \leq 0.01$. TAZ, transcriptional coactivator with PDZ-binding motif.

(Fig. 1D; lower graph). Thus, as previously observed in other cancer types, TAZ mRNA and protein were overexpressed in prostate cancer tissues.
TAZ knockdown in PC3 cells inhibits prostate cancer cell viability. Comparison of TAZ expression levels in multiple prostate cancer cell lines showed that TAZ mRNA was 
A

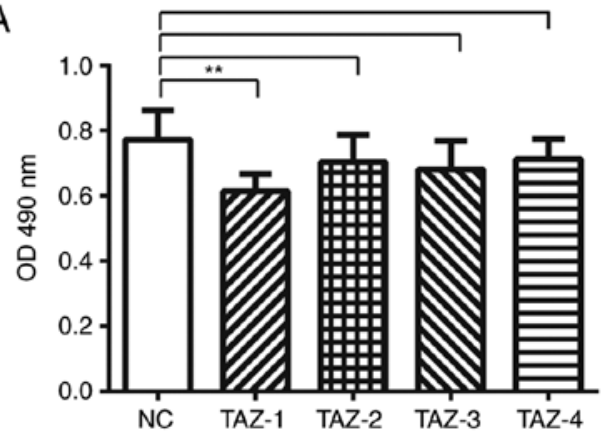

B

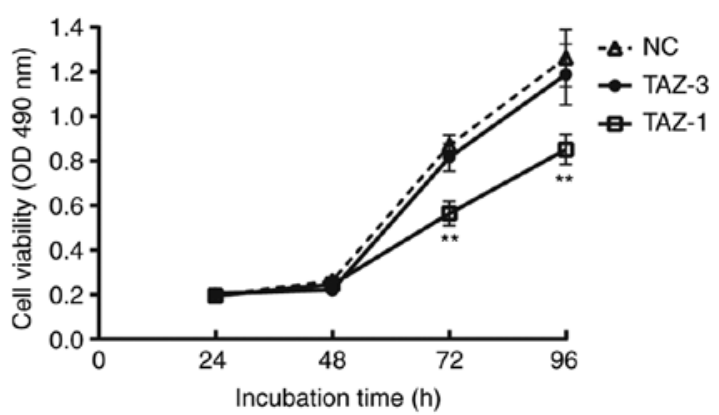

Figure 3. TAZ knockdown leads to inhibition of PC3 cell viability. (A) MTT cell viability assays were performed $60 \mathrm{~h}$ after cell transfection with 4 species of TAZ shRNA. A significant inhibition of cell viability was observed in cells transfected with TAZ-1 compared with the control group. (B) After cell transfection with the shRNAs, TAZ-1 but not TAZ-3 was found to significantly inhibited cell viability at 72 and $96 \mathrm{~h}$ post-transfection, respectively. ** $\mathrm{P} \leq 0.01$, compared with the negative control (NC) group. TAZ, transcriptional coactivator with PDZ-binding motif.

A
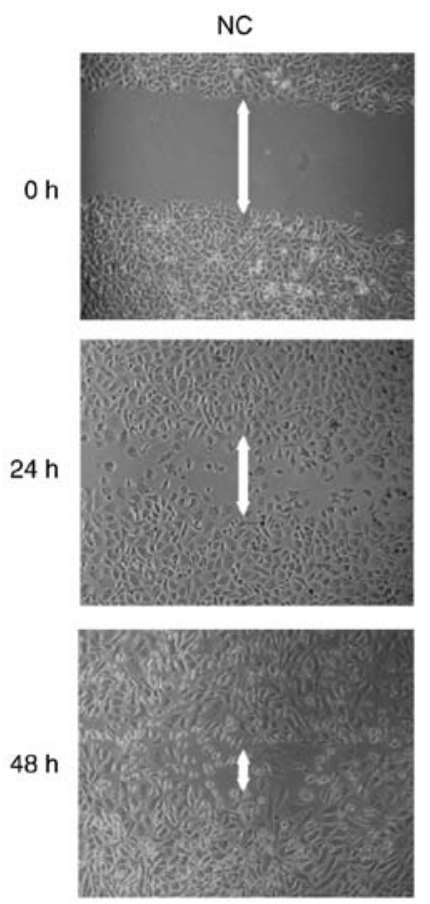

C

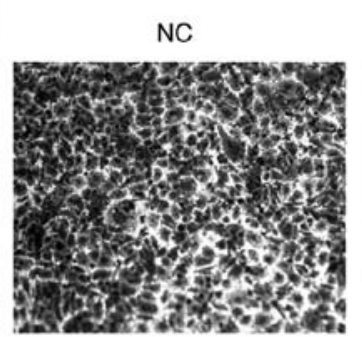

TAZ-1
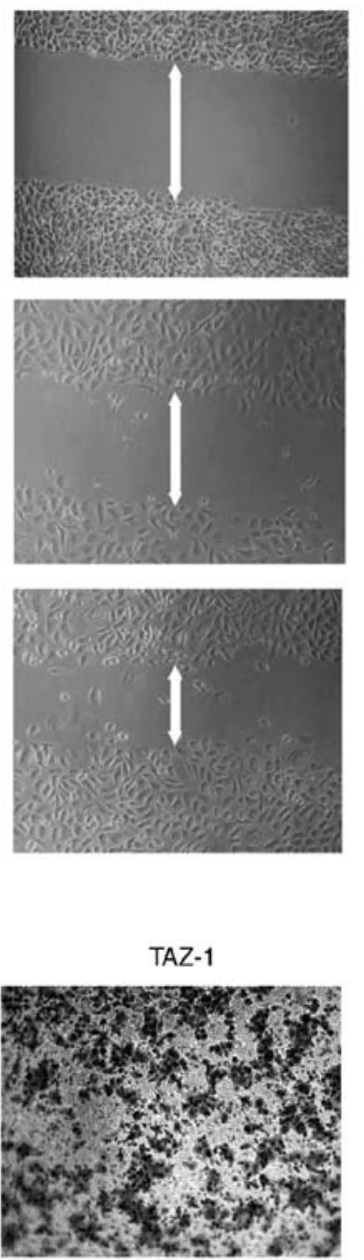

\section{B}
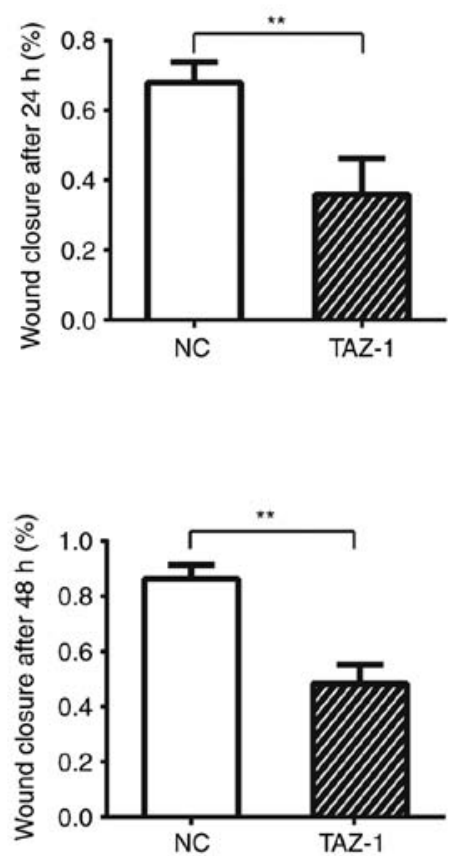

D

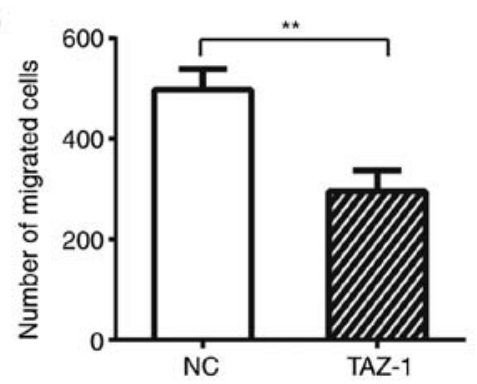

Figure 4. TAZ knockdown inhibits PC3 cell migration. (A) Cells were transfected with control (NC) or TAZ-1 shRNAs. At 24 h post-transfection, cell cultures were scratched, and wound closures were measured at 0,24 and $48 \mathrm{~h}$ after scratching. (B) A significantly wider gap (reduced wound closure) was observed in cell cultures transfected with TAZ-1 shRNA compared with the cultures transfected with the control shRNA (NC) at 24 (upper panel) and 48 h (lower panel) after scratch, indicating decreased cell migration. (C) Transwell assays were performed at $36 \mathrm{~h}$ after transfection with TAZ-1 or control shRNA (NC). (D) A reduced cell migration was observed in cells transfected with TAZ-1 shRNA in comparison with the control (NC) cells, indicating that TAZ knockdown significantly inhibited cell migration. ${ }^{* *} \mathrm{P} \leq 0.01$. TAZ, transcriptional coactivator with PDZ-binding motif.

highly expressed in the PC3 cell line (Fig. 2A), which was subsequently used for the knockdown experiments. Four short hairpin RNA (shRNA) species and a nonspecific control RNA
(NC) were designed and subcloned into a plasmid to be used in the transfection experiments. TAZ mRNA and protein levels were determined by real-time PCR and western blot 

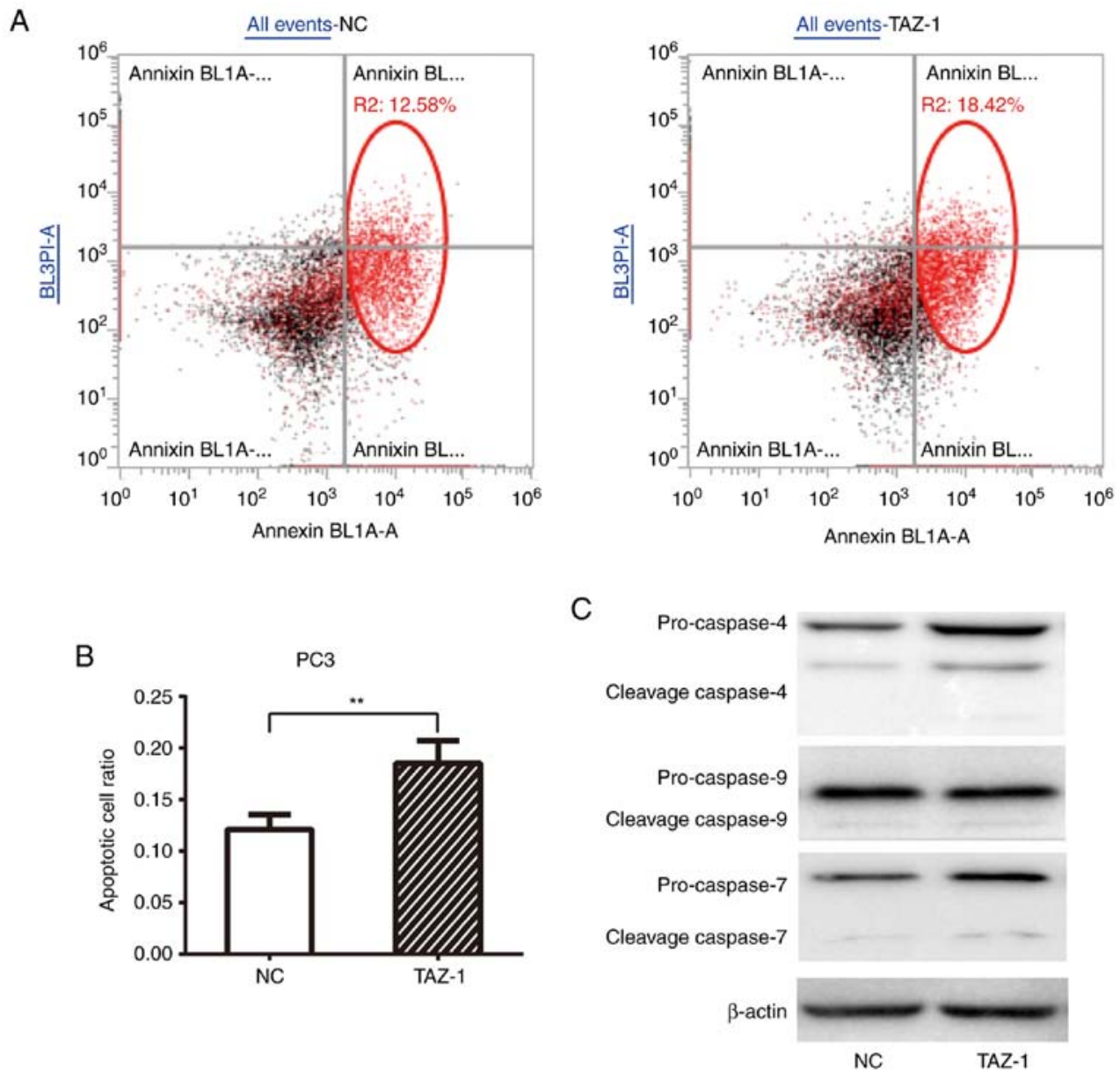

Figure 5. TAZ knockdown induces apoptosis in prostate cancer PC3 cells. (A) PC3 cells were transfected with control (NC) or TAZ-1 shRNAs. Flow cytometric analysis was performed at $48 \mathrm{~h}$ post-transfection, and cell death was compared. (B) Statistical analysis showed an increased apoptosis in cells transfected with TAZ-1 in comparison with NC group. (C) Western blotting results indicated that at $72 \mathrm{~h}$ of post-transfection with TAZ-1 shRNA, the expression as well as the cleavage of caspase- 4 and caspase-7, but not caspase-9, were greatly increased, confirming the apoptotic effects of TAZ knockdown in PC3 cells. ${ }^{* *} \mathrm{P} \leq 0.01$. TAZ, transcriptional coactivator with PDZ-binding motif.

assays, respectively. While two shRNAs (TAZ-2 and TAZ-4) had no significant effect on TAZ expression, TAZ-1 and TAZ-3 effectively inhibited TAZ expression (Fig. 2B and C). Following cell transfection with the four vectors containing different TAZ shRNAs, an MTT cell viability assay was performed (Fig. 3A). The results indicated that the knockdown of TAZ expression by TAZ-1 led to a significant inhibition of cell viability compared with the non-specific control group. In additional experiments, after transfection with TAZ-1 and TAZ-3 vectors, MTT assays were performed at different time points to establish cell viability curves (Fig. 3B). TAZ-1, but not TAZ-3 or the non-specific control RNA, caused a significant inhibition in cell viability at $72-$ and $96-\mathrm{h}$ post-transfection. Although both TAZ-1 and TAZ-3 appeared to be effective for TAZ knockdown on the mRNA level, for some unknown reasons, only TAZ-1 showed effects in the cell viability experiment. Therefore, TAZ-1 was used for subsequent experiments.

TAZ knockdown inhibits PC3 cell migration. We performed a wound scratch assay to determine the function of TAZ in cell migration of prostate cancer cells. At $48 \mathrm{~h}$ after transfection with TAZ-1 and control RNA vectors, wound scratches were made in cell cultures. Cell culture images were captured, and the scratch gap was measured. At 24- and 48-h post-transfection, the cell culture with TAZ knockdown showed a significantly wider gap than the control culture (Fig. 4A and B). After $48 \mathrm{~h}$, the control culture showed near closure of the gap, whereas the TAZ knockdown cell culture had a gap measured approximately $50 \%$ of the original width (Fig. 4B). Similarly, results of the Transwell assay at 36-h post-transfection revealed that TAZ knockdown led to a significant inhibition of cell migration (Fig. 4C).

TAZ knockdown induces cell apoptosis in the PC 3 and $D U$-145 cell lines. To further clarify the biological functions of TAZ, cell apoptosis assays were performed. At 48-h post-transfection, PC3 and DU-145 cells were collected, fixed, and stained with a FITC-labelled Annexin antibody and with PI. Flow cytometric analysis was carried out to compare cell death between the TAZ-1 treatment group and the control group. The results showed that TAZ knockdown in these cells led to a significant increase in cell apoptosis in the PC3 (Fig. 5A and B) and DU-145 cell lines (Fig. 6A and B). To investigate the mechanism of apoptosis, we performed western blot analysis of caspase- $4,-9$ and -7 , and found that the protein expression levels and cleavage products of caspase- 4 , and -7 
A
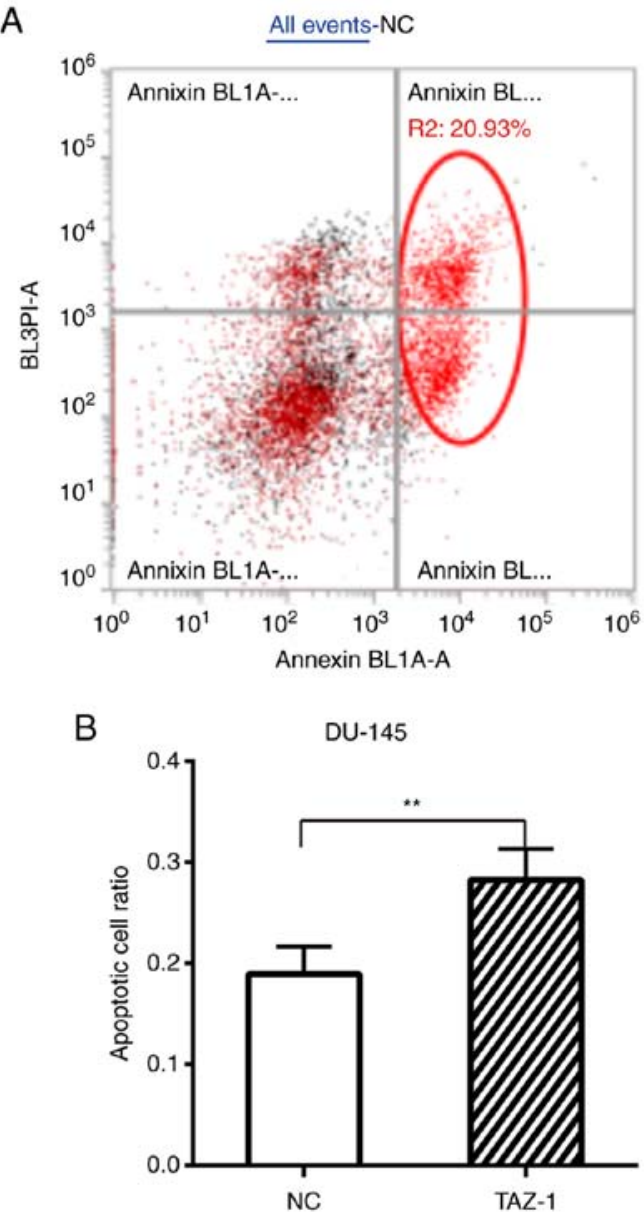

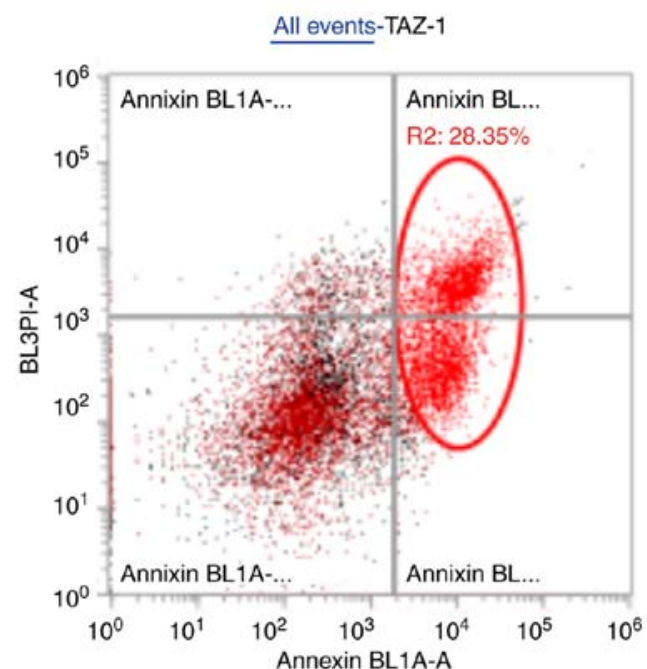

C

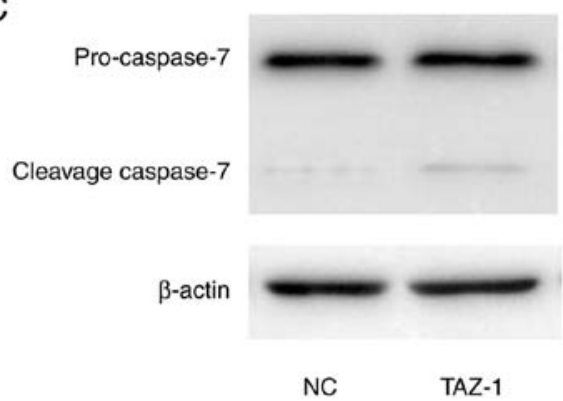

Figure 6. TAZ knockdown induces apoptosis in prostate cancer DU-145 cells. (A) DU-145 cells were transfected with control (NC) or TAZ-1 shRNAs. At 48-h post-transfection, flow cytometry was performed and cell death was compared. (B) Statistical analysis showed an increased apoptosis in cells transfected with TAZ-1 in comparison with the NC group. (C) Western blotting results indicated that at $72 \mathrm{~h}$ post-transfection with TAZ-1 shRNA, the cleavage of caspase-7 was increased, confirming the apoptotic effects of TAZ knockdown in DU-145 cells. ${ }^{* *} \mathrm{P} \leq 0.01$. TAZ, transcriptional coactivator with PDZ-binding motif.

(but not caspase-9) were obviously elevated following TAZ knockdown in the PC3 cell line (Fig. 5C). In regards to the DU-145 cell line, after TAZ knockdown, the protein expression level and cleavage products of caspase-7 were also obviously increased (Fig. 6C). These results indicated that the reduction in TAZ led to cell apoptosis through the exogenous apoptotic pathway mediated by caspase-7 cleavage.

TAZ knockdown inhibits xenograft tumor growth. PC3 cells were transfected with TAZ-1 or control vectors, and stably transfected transfection clones were selected by treatment with puromycin treatment for 21 days. Stably transfected cells were mixed with Matrigel matrix and were injected subcutaneously into the right (TAZ-1) and left (NC) rear flanks of 5 male nude mice. Tumor growth was monitored by measurement of tumor volume for the TAZ knockdown and control groups. Tumor volumes were calculated, and tumor growth curves were established. It was observed that TAZ-1 stable transfectants and control cells were capable of developing tumor masses after inoculation. However, TAZ knockdown transfectants exhibited significant decreased tumor volume at 12 days and thereafter (Fig. 7A and B). On the 16th day, mice were sacrificed and tumors were dissected and weighed. The mean tumor weight of the TAZ-1 group was significantly lower than that of the control group (Fig. 7C). These findings indicated that TAZ knockdown substantially affected tumor growth, highlighting a significant role for TAZ-1 in PC3 cell malignant behavior in vivo.

\section{Discussion}

TAZ (transcriptional coactivator with PDZ-binding motif), which is also known as WW domain-containing transcription regulator 1 (WWTR1) is known to be overexpressed in multiple types of tumors including breast cancer, lung cancer and ovarian malignancies $(10,13,16)$. It was reported that increased TAZ expression is associated with the malignant features of tumors and the poor survival of patients. Zhang et al observed that high TAZ expression in retinoblastoma lesions was correlated with a larger tumor base, regional lymph node metastasis, lower tumor cell differentiation, and shorter overall survival (OS) as well as progression-free survival (PFS) (15). In another study, Wei et al examined 214 gastric cardia adenocarcinomas and found that TAZ expression was inversely correlated with cumulative survival. The authors proposed that TAZ, which is a Hippo signaling effector, could be a metastatic biomarker for 


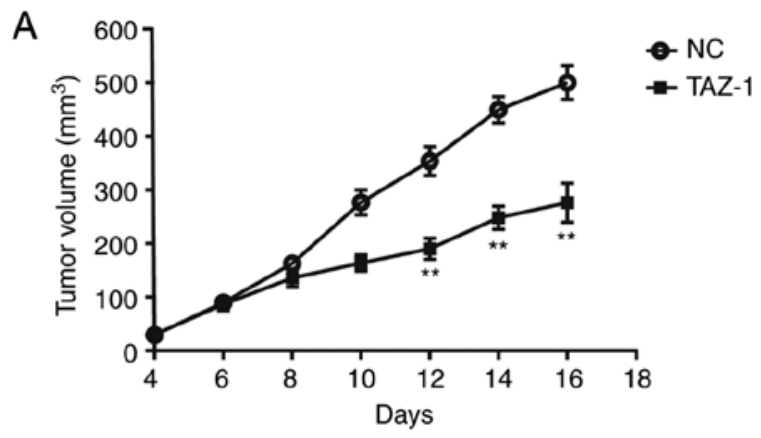

B
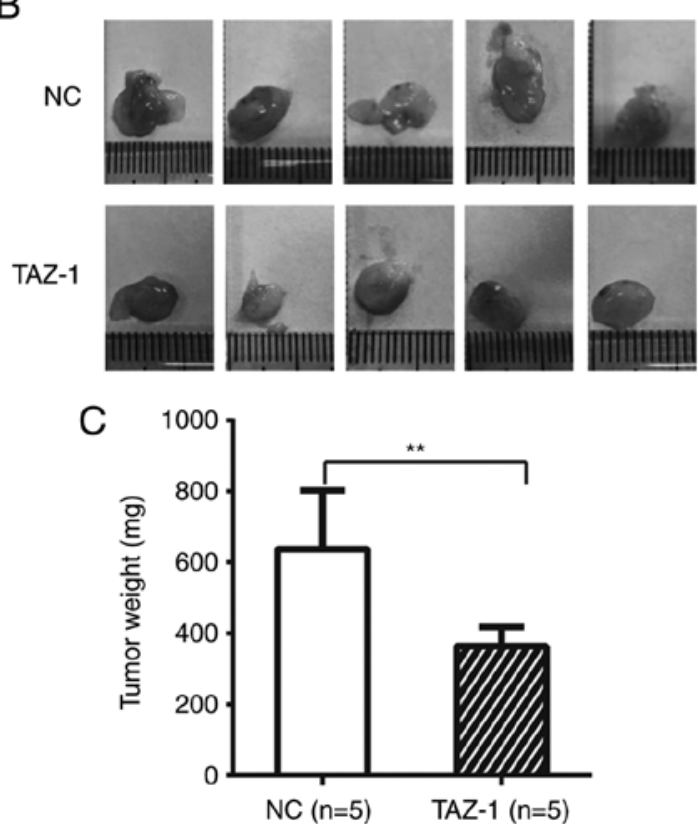

Figure 7. TAZ knockdown inhibits xenograft tumor growth. (A) PC3 cells transfected with control (NC) or TAZ-1 shRNAs were injected subcutaneously into the rear flanks of nude mice. The tumor diameters were measured and the tumor volumes $(\mathrm{V})$ were estimated every 2 days using the equation $\mathrm{V}=0.5 \times \mathrm{a} \mathrm{x} \mathrm{b}^{2}$ (a, length; $\mathrm{b}$, width). Significantly decreased tumor volumes were observed for cells transfected with TAZ-1 shRNA. (B) Sixteen days after tumor cell inoculation, the mice were sacrificed and tumous were dissected and weighted. (C) Statistical analysis of tumor weight showed that the mean tumor weight of the TAZ knockdown group was markedly lower than that of the control (NC) group. ${ }^{* *} \mathrm{P} \leq 0.01$. TAZ, transcriptional coactivator with PDZ-binding motif.

gastric cardia adenocarcinoma (21). Our immunohistochemistry results indicated that higher grade PCa tissues expressed significantly increased levels of TAZ, suggesting that the function of TAZ is associated with the malignant behaviors of prostate cancer. This observation also prompted us to investigate the function of TAZ in prostate cancer cells.

Accumulated data supported that TAZ may participate in the regulation of cell proliferation, migration and apoptosis. Previously, in vitro studies showed that in the gastric cancer AGS cell line, shRNA-mediated knockdown of TAZ resulted in impaired cell migration (21). In addition, TAZ overexpression triggered a loss of epithelial morphology $(22,23)$, promoted cell viability, and supported anchorage-independent growth $(7,14)$. In the present study, we demonstrated that shRNA-mediated TAZ knockdown in PC3 cells inhibited cell viability and reduced cell migration, suggesting a positive role for TAZ in cell proliferation and cell mobility. This observation is reminiscent of the close association of TAZ expression levels with high pathological grade of prostate cancers. Both TAZ and YAP contain the WW domain that is known to mediate protein-protein interactions. TAZ alone or TAZ/YAP complex is able to interact with TEAD1/4 in the nucleus to modulate the latter's DNA binding and transcriptional activity $(24,25)$. Hayashi et al performed quantitative PCR in HepG2 cells and found that siRNA-mediated TAZ knockdown resulted in decreased expression levels of cysteine-rich angiogenic inducer 61 (CYR61) and connective tissue growth factor (CTGF) mRNA (26). CTGF and Cyr61 proteins promote cell migration and inhibit cell apoptosis $(27,28)$. Genetic ablation of TAZ induces HepG2 liver cancer cell apoptosis by activating the CaMKII/ MIEF1 signaling pathway (29). In CCLP-1, an intrahepatic cholangiocarcinoma cell line, TAZ knockdown promoted P53 expression and induced cell apoptosis $(30,31)$. It was also reported that phosphorylation of YAP and TAZ by SRC/ ABL tyrosine kinase could enhance their interaction with P53 in mammary stem cells (32). Thus, as observed in this and previous studies that one important function of TAZ could be the promotion of cancer progression, which is most likely achieved by a divergent set of mechanisms. However, these mechanisms were described in non-prostate cancer cells, and further investigation on the upstream and downstream events in prostate cancers are required for a complete picture of the TAZ regulatory pathway leading to changes in cell migration and apoptosis.

Our in vivo experiments using a mouse subcutaneous xenograft model demonstrated that TAZ knockdown in PC3 cells resulted in a lower tumor growth rate, suggesting that tumor growth relied on a sufficient level of TAZ. This observation is consistent with the findings that TAZ overexpression correlated with a high grade of prostate cancer. Previous studies indicated that when TAZ expression was knocked down by siRNA treatment of HepG2 cells, caspase-3 cleavage was increased (33). Our findings indicated that TAZ knockdown led to increased expression and cleavage of caspase-4. Since both caspase-3 and -7 are common executors of cell apoptosis, our observation is consistent with previous research. Moreover, our results showing the increased expression and cleavage of caspase- 4 indicated that TAZ knockdown induced cell apoptosis through the activation of the exogenous apoptotic pathway.

Our comparison of four prostate cancer cell lines PC3, DU-145, LNCaP and 22RV1, and the normal prostate epithelial cell line RWPE-1 showed that these cells express varied TAZ mRNA levels, with PC3 cells expressing a relatively higher level of TAZ mRNA. However, we could not established if the in vivo growth of these cell lines was related to their TAZ levels since xenograft growth curves were not determined for all cancer cell lines except for PC3. Similarly, although knockdown experiments showed a positive correlation between tumor growth and TAZ expression levels, effects of TAZ overexpression on tumor growth was not examined. Secondly, analysis of cell apoptosis following TAZ knockdown was determined only in PC3 and DU-145 cell lines. Thirdly, although we determined the TAZ expression levels in human prostate cancer tissues and found a direct correlation between TAZ levels and tumor grade, TAZ expression levels were not determined in the xenograft tissues. These disadvantages to a 
certain extent limited the extrapolation of our observation to a broader scope, e.g., other prostate cancer cells.

Taken together, the present study demonstrated that TAZ plays a critical role in the viability, migration, and apoptosis of prostate cancer cells. The significant increase in TAZ expression in prostate cancers, especially the high TAZ levels in high Gleason score (H-GL) tissues, suggested that TAZ could be a useful biomarker for the diagnosis and/or prognosis of prostate cancer patients. Since TAZ tends to be overexpressed in high-grade cancers, and since sufficient TAZ levels appear to be required for the vitality and functions of prostate cancer cells, TAZ targeting could be a novel therapeutic strategy for treating the most lethal subgroup of prostate malignancies.

\section{Acknowledgements}

The authors would like to thank the Jiangnan University for providing supervision and the animal facility to our research group to complete the mouse xenograft experiments.

\section{Funding}

The present study was supported by the Jiangsu medical leading talents Project (CXTDA2017047), the Jiangsu Provincial Key Research and Development Program (Social Development) Project (BE2018629), and research funding from The Affiliated Wuxi Maternity and Child Care Health Hospital of Nanjing Medical University.

\section{Availability of data and materials}

The datasets used and/or analyzed during the present study are available from the corresponding author on reasonable request.

\section{Authors' contributions}

NF and SWJ designed and supervised the research project. $\mathrm{ML}, \mathrm{QH}$ and CB performed the western blotting, cell culture experiments, and data analysis. JG collected and prepared the tissue samples. ML and HW carried out the cell culture and Transwell assays. SWJ and ML prepared the manuscript. All authors have read and approved the manuscript, and agreed to be accountable for all aspects of the research.

\section{Ethics approval and consent to participate}

This study was approved by the Human Research Ethics Committee of the Affiliated Wuxi No. 2 People's Hospital of Nanjing Medical University (no. 20170719). All participants signed informed consent prior to enrollment. The usage of mice for the xenograft experiment was approved by the Institutional Animal Ethics Committee of Jiangnan University.

\section{Patient consent for publication}

Not applicable.

\section{Competing interests}

The authors declare that they have no competing interests.

\section{References}

1. Chen W, Zheng R, Baade PD, Zhang S, Zeng H, Bray F, Jemal A, Yu XQ and He J: Cancer statistics in China, 2015. CA Cancer J Clin 66: 115-132, 2016.

2. Pullar B and Shah N: Prostate cancer. Surgery (Oxford) 34: 505-511, 2016.

3. Chaitoff A, Killeen TC and Nielsen C: Men's health 2018: BPH, prostate cancer, erectile dysfunction, supplements. Cleve Clin J Med 85: 871-880, 2018.

4. Kanai F, Marignani PA, Sarbassova D, Yagi R, Hall RA, Donowitz M, Hisaminato A, Fujiwara T, Ito Y, Cantley LC and Yaffe MB: TAZ: A novel transcriptional co-activator regulated by interactions with 14-3-3 and PDZ domain proteins. EMBO J 19: 6778-6791, 2000.

5. Santucci M, Vignudelli T, Ferrari S, Mor M, Scalvini L, Bolognesi ML, Uliassi E and Costi MP: The Hippo pathway and YAP/TAZ-TEAD protein-protein interaction as targets for regenerative medicine and cancer treatment. J Med Chem 58: 4857-4873, 2015.

6. Brusatin G, Panciera T, Gandin A, Citron A and Piccolo S: Biomaterials and engineered microenvironments to control YAP/ TAZ-dependent cell behaviour. Nat Mater 17: 1063-1075, 2018.

7. Meng Z, Moroishi T and Guan KL: Mechanisms of hippo pathway regulation. Genes Dev 30: 1-17, 2016.

8. Yuen HF, McCrudden CM, Huang YH, Tham JM, Zhang X, Zeng Q, Zhang SD and Hong W: TAZ expression as a prognostic indicator in colorectal cancer. PLoS One 8: e54211, 2013.

9. Bhat KP, Salazar KL, Balasubramaniyan V, Wani K, Heathcock L, Hollingsworth F, James JD, Gumin J, Diefes KL, Kim SH, et al: The transcriptional coactivator TAZ regulates mesenchymal differentiation in malignant glioma. Genes Dev 25: 2594-2609, 2011.

10. Chen G, Xie J, Huang P and Yang Z: Overexpression of TAZ promotes cell proliferation, migration and epithelial-mesenchymal transition in ovarian cancer. Oncol Lett 12: 1821-1825, 2016.

11. Jeong GO, Shin SH, Seo EJ, Kwon YW, Heo SC, Kim KH, Yoon MS, Suh DS and Kim JH: TAZ mediates lysophosphatidic acid-induced migration and proliferation of epithelial ovarian cancer cells. Cell Physiol Biochem 32: 253-263, 2013.

12. Lei QY, Zhang H, Zhao B, Zha ZY, Bai F, Pei XH, Zhao S, Xiong Y and Guan KL: TAZ promotes cell proliferation and epithelial-mesenchymal transition and is inhibited by the hippo pathway. Mol Cell Biol 28: 2426-2436, 2008.

13. Chan SW, Lim CJ, Guo K, Ng CP, Lee I, Hunziker W, Zeng Q and Hong W: A role for TAZ in migration, invasion, and tumorigenesis of breast cancer cells. Cancer Res 68: 2592-2598, 2008.

14. Moroishi T, Hansen CG and Guan KL: The emerging roles of YAP and TAZ in cancer. Nat Rev Cancer 15: 73-79, 2015.

15. Zhang Y, Xue C, Cui H and Huang Z: High expression of TAZ indicates a poor prognosis in retinoblastoma. Diagn Pathol 10: 187, 2015.

16. Malik SA, Khan MS, Dar M, Hussain MU and Mudassar S: TAZ is an independent prognostic factor in non-small cell lung carcinoma: Elucidation at protein level. Cancer Biomark 18: 389-395, 2017.

17. Wang Y, Xu X, Maglic D, Dill MT, Mojumdar K, Ng PK, Jeong KJ, Tsang YH, Moreno D, Bhavana VH, et al: Comprehensive molecular characterization of the hippo signaling pathway in cancer. Cell Rep 25: 1304-1317, 2018.

18. Mills GB and Moolenaar WH: The emerging role of lysophosphatidic acid in cancer. Nat Rev Cancer 3: 582-591, 2003.

19. Na Y, Ye Z, Sun Y and Sun G: Guidelines for Diagnosis and Treatment of Urology in China (2014). People's Medical Publishing House, 2013. https://www.dxy.cn/bbs/newweb/pc/post/28238341.

20. Livak KJ and Schmittgen TD: Analysis of relative gene expression data using real-time quantitative PCR and the 2(-Delta DeltaC(T)) method. Methods 25: 402-408, 2001.

21. Wei J, Wang L, Zhu J, Sun A, Yu G, Chen M, Huang P, Liu H, Shao G, Yang W and Lin Q: The Hippo signaling effector WWTR1 is a metastatic biomarker of gastric cardia adenocarcinoma. Cancer Cell Int 19: 74, 2019.

22. Liu N, Mei L, Fan X, Tang C, Ji X, Hu X, Shi W, Qian Y, Hussain M, Wu J, et al: Phosphodiesterase 5/protein kinase G signal governs stemness of prostate cancer stem cells through hippo pathway. Cancer Lett 378: 38-50, 2016.

23. Dogruluk T, Tsang YH, Espitia M, Chen F, Chen T, Chong Z, Appadurai V, Dogruluk A, Eterovic AK, Bonnen PE, et al: Identification of variant-specific functions of PIK3CA by rapid phenotyping of rare mutations. Cancer Res 75: 5341-5354, 2015. 
24. Zhao B, Ye X, Yu J, Li L, Li W, Li S, Yu J, Lin JD, Wang CY, Chinnaiyan AM, et al: TEAD mediates YAP-dependent gene induction and growth control. Genes Dev 22: 1962-1971, 2008.

25. Holden JK and Cunningham CN: Targeting the hippo pathway and cancer through the TEAD family of transcription factors. Cancers (Basel) 10: 10, 2018.

26. Hayashi H, Higashi T, Yokoyama N, Kaida T, Sakamoto K, Fukushima Y, Ishimoto T, Kuroki H, Nitta H, Hashimoto D, et al: An imbalance in TAZ and YAP expression in hepatocellular carcinoma confers cancer stem cell-like behaviors contributing to disease progression. Cancer Res 75: 4985-4997, 2015.

27. Sakai Y, Balam TA, Kuroda S, Tamamura N, Fukunaga T, Takigawa $\mathrm{M}$ and Takano-Yamamoto T: CTGF and apoptosis in mouse osteocytes induced by tooth movement. J Dent Res 88: 345-350, 2009

28. Kireeva ML, Mo FE, Yang GP and Lau LF: Cyr61, a product of a growth factor-inducible immediate-early gene, promotes cell proliferation, migration, and adhesion. Mol Cell Biol 16: 1326-1334, 1996.
29. Hou Y, Lan C, Kong Y, Zhu C, Peng W, Huang Z and Zhang C: Genetic ablation of TAZ induces HepG2 liver cancer cell apoptosis through activating the CaMKII/MIEF1 signaling pathway. Onco Targets Ther 12: 1765-1779, 2019.

30. Xiao H, Tong R, Yang B, Lv Z, Du C, Peng C, Ding C, Cheng S, Zhou L, Xie H, et al: TAZ regulates cell proliferation and sensitivity to vitamin D3 in intrahepatic cholangiocarcinoma. Cancer Lett 381: 370-379, 2016.

31. Furth N, Aylon Y and Oren M: p53 shades of hippo. Cell Death Differ 25: 81-92, 2018.

32. Su X, Napoli M, Abbas HA, Venkatanarayan A, Bui NHB, Coarfa C, Gi YJ, Kittrell F, Gunaratne PH, Medina D, et al: TAp63 suppresses mammary tumorigenesis through regulation of the hippo pathway. Oncogene 36: 2377-2393, 2017.

33. Ji K, Lin K, Wang Y, Du L, Xu C, He N, Wang J, Liu Y and Liu Q: TAZ inhibition promotes IL-2-induced apoptosis of hepatocellular carcinoma cells by activating the JNK/F-actin/ mitochondrial fission pathway. Cancer Cell Int 18: 117, 2018. 